\title{
Economisez des milliers de francs dans la LPP
}

\section{Vérifiez votre caisse de pension actuelle!}

\section{D'énormes différences}

Les écarts des prix/prestations des prestataires de caisses de pension sur le marché sont considérables. Surtout les points suivants doivent être observés avec attention:

- niveau des coûts de frais d'administration et des risques;

- taux d'intérêt du capital-vieillesse du régime obligatoire et sur-obligatoire;

- taux de conversion de la rente.

\section{Comparez, cela en vaut la peine!}

Comme vous le constatez dans l'exemple ci-dessous, une analyse comparative gratuite de votre prévoyance professionnelle par un conseiller FMH Insurance Services est indispensable:

\begin{tabular}{|c|c|c|c|}
\hline Homme, âge 60 & Avant l'analyse & Après I'analyse & $\begin{array}{l}\text { Votre avantage } \\
\text { pendant } 5 \text { ans }\end{array}$ \\
\hline Revenu assuré & CHF 220000 & CHF 220000 & \\
\hline Capital à la retraite taux $(2,50 \%)$ inclus & CHF 446600 & CHF 470500 & 23900 \\
\hline Rente invalidité & 55000 & 55000 & \\
\hline Rente de conjoint & 33000 & 33000 & \\
\hline Cotisations annuelles & 53800 & 47800 & 30000 \\
\hline \multicolumn{3}{|c|}{ L'économie réalisée pendant la période de 5 ans (à l'âge de 65 ans) } & 53900 \\
\hline
\end{tabular}

\section{Délai de résiliation: jusqu'au 30 juin 2007!}

De nombreux contrats de prévoyance ne peuvent être résiliés que 6 mois avant la fin de l'année en cours. Retournez-nous le talon ci-joint encore aujourd'hui afin d'analyser votre contrat actuel et de vous envoyer une offre.

\section{Profitez de notre offre}

Vos besoins sont au centre de nos préoccupations. Notre nom vous garantit le conseil professionnel de nos conseillers qui possèdent une longue expérience dans le domaine des assurances, des produits bancaires et financiers. Grâce à notre indépendance nous pouvons vous offrir les meilleurs produits adaptés à vos besoins.

\section{Talon réponse}

Prénom / Nom

Adresse

NPA / Lieu

Date de naissance

Téléphone privé/cabinet

Atteignable le plus facilement (heure)

Adresse e-mail

J Je désire un conseil personnalisé en LPP (prière de joindre le certificat d'assurance de votre caisse de pension actuelle).

Je m'intéresse à:

\begin{tabular}{ll} 
Planification de la retraite & O Planification financière/impôts \\
O 3 $^{\mathrm{e}}$ pilier a & O Protection juridique \\
C Caisse maladie & O Responsabilité civile prof. \\
\hline
\end{tabular}

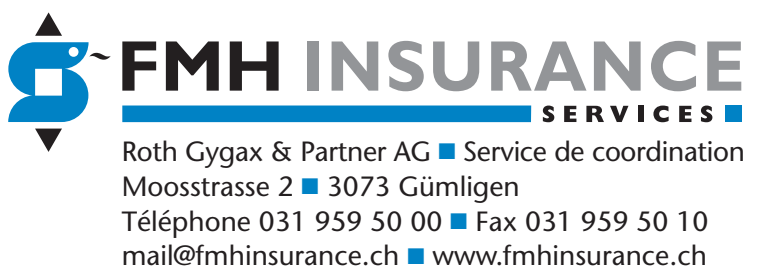

